\title{
Electron effective mass and mobility in heavily doped $n$-GaAsN probed by Raman scattering
}

\author{
J. Ibáñez, ${ }^{1}$ R. Cuscó, ${ }^{1}$ E. Alarcón-Lladó, ${ }^{1}$ L. Artús, ${ }^{1, a)}$ A. Patanè, ${ }^{2}$ D. Fowler, ${ }^{2}$ L. Eaves, ${ }^{2}$ \\ K. Uesugi, ${ }^{3}$ and I. Suemune ${ }^{4}$ \\ ${ }^{1}$ Institut Jaume Almera, Consell Superior d'Investigacions Científiques (CSIC), Lluis Solé i Sabarís s.n., \\ 08028 Barcelona, Catalonia, Spain \\ ${ }^{2}$ School of Physics and Astronomy, University of Nottingham, NG7 2RD Nottingham, United Kingdom \\ ${ }^{3}$ Faculty of Engineering, Muroran Institute of Technology, Mizumotocho, Muroran, \\ Hokkaido 050-8585, Japan \\ ${ }^{4}$ Research Institute for Electronic Science (RIES), Hokkaido University, Sapporo 060-0812, Japan
}

(Received 5 December 2007; accepted 18 March 2008; published online 27 May 2008)

We investigate inelastic light scattering by longitudinal optic phonon-plasmon coupled modes (LOPCMs) in a series of heavily Se-doped, $n$-type $\mathrm{GaAs}_{1-x} \mathrm{~N}_{x}$ epilayers with $x<0.4 \%$. We perform a line shape analysis of the LOPCM spectra to estimate the optical effective mass, $m_{\mathrm{opt}}^{*}$, and the scattering time of the conduction electrons in GaAsN. We use these results to evaluate an effective carrier mobility for our samples. The values thus obtained, which we compare with measured electron Hall mobilities, indicate that the $x$-dependence of the mobility in $\mathrm{GaAs}_{1-x} \mathrm{~N}_{x}$ is dominated by the scattering time, rather than by the variation of the electron effective mass. The Raman analysis yields $m_{\mathrm{opt}}^{*}$ values that are lower than those obtained from the band anticrossing model.

(C) 2008 American Institute of Physics. [DOI: 10.1063/1.2927387]

\section{INTRODUCTION}

${ }^{1}$ Dilute nitride compounds, in particular GaAsN and GaInAsN, have attracted much interest over the last decade because of their remarkable physical properties and their potential for device applications in long-wavelength optoelectronics. ${ }^{1}$ It has been established that the incorporation of a few atomic percent of $\mathrm{N}$ into the $\mathrm{Ga}(\mathrm{In})$ As alloy system leads to a substantial band-gap reduction, as well as to the splitting of the conduction band of the III-V matrix into two highly nonparabolic subbands, $E_{+}$and $E_{-}{ }^{2-5}$ The simple two-level band anticrossing (BAC) model $^{2}$ provides a reasonably accurate description of the dependence on $\mathrm{N}$ content of the energies of $E_{+}$and $E_{-}$. Also, it predicts a substantial increase in the effective mass $\left(m_{e}^{*}\right)$ for the lower energy subband $E_{-}$relative to the III-V matrix. ${ }^{6,7}$ However, a complete description of the band structure of dilute nitrides requires detailed calculations of the localized electronic states introduced by $\mathrm{N}$ and their interaction with the extended conduction states of the host matrix. ${ }^{4,5}$ While magneto-tunneling measurements have revealed the admixed character of the $\mathrm{N}$-induced states and the anticrossing behavior of the $E_{-}$and $E_{+}$subbands in $\mathrm{GaAs}_{1-x} \mathrm{~N}_{x}$ quantum wells, ${ }^{8,9}$ optical and transport techniques have confirmed the increase of $m_{e}^{*}$ of conduction electrons in both GaAsN and GaInAsN. ${ }^{6,7,10-13}$

Most of the optical experiments to determine $m_{e}^{*}$ in dilute $\mathrm{Ga}(\mathrm{In}) \mathrm{AsN}$ were performed on undoped material. As a consequence, the $m_{e}^{*}$ values obtained in those experiments correspond to the bottom of the $E_{-}$conduction subband $\left(m_{e 0}^{*}\right)$. In order to use optical techniques to investigate the conduction band (CB) dispersion of $\mathrm{Ga}(\mathrm{In}) \mathrm{AsN}$ and determine $m_{e}^{*}$ far above the zone-center minimum of $E_{-}$, heavily

${ }^{a)}$ Electronic mail: lartus@ija.csic.es. doped $n$-type samples are required. Studies on highly doped material tend to confirm the validity of the BAC model as a description of the energy-wave vector dispersion, $E(k)$, in the case of the GaInAsN quaternary alloy. ${ }^{6,7}$ In contrast, magnetophotoluminescence measurements on undoped $\mathrm{GaAs}_{1-x} \mathrm{~N}_{x}$ suggest that the BAC model underestimates $m_{e 0}^{*}$ for $x$ $\geq 0.1 \%,{ }^{12}$ while experimental studies probing $m_{e}^{*}$ in this compound at energies above the CB minimum are presently lacking. Additional experiments are therefore necessary to determine how $E(k)$ and $m_{e}^{*}(k)$ depend on $\mathrm{N}$ content both in GaAsN and GaInAsN.

Raman scattering by longitudinal optical (LO) phononplasmon coupled modes (LOPCMs) has been widely reported in the literature as a method for determining the freecarrier concentration in polar semiconductors. ${ }^{14-19}$ Conversely, the investigation of LOPCMs can also be used to optically probe the $\mathrm{CB}$ dispersion of the compound under study if the carrier concentration, $N_{e}$, is known from independent measurements, since the frequency of the LOPCMs strongly depends on $m_{e}^{*}$. It should be recalled that optical experiments probe the optical effective mass of the carriers, $m_{\mathrm{opt}}^{*}$, which exhibits a marked $N_{e}$-dependence in nonparabolic bands. $^{20,21}$

In the present work, we use Raman scattering to investigate the LOPCMs in a series of heavily doped $n$-type $\mathrm{GaAs}_{1-x} \mathrm{~N}_{x}$ epilayers $(0 \%<x<0.4 \%)$. From the line shape analysis of the LOPCM spectra, we estimate $m_{\mathrm{opt}}^{*}$ and the scattering time, $\tau$, of conduction electrons in our samples. We use the values of $m_{\mathrm{opt}}^{*}$ and $\tau$ to obtain an effective electron mobility which we compare with the Hall effect mobility. We find that the values of $m_{\mathrm{opt}}^{*}$ are lower than those predicted by the BAC model. Our analysis suggests that the 
TABLE I. List of $n$-type Se-doped $\mathrm{GaAs}_{1-x} \mathrm{~N}_{x}$ epilayers studied in this work.

\begin{tabular}{ccccccc}
\hline \hline Sample & $x$ & $N_{e}\left(\mathrm{~cm}^{-3}\right)$ & $\mu_{\text {Hall }}\left(\mathrm{cm}^{2} / \mathrm{Vs}\right)$ & $m_{\mathrm{opt}}^{*} / m_{e}$ & $\Gamma_{e}\left(\mathrm{~cm}^{-1}\right)$ & $\mu_{\text {Raman }}\left(\mathrm{cm}^{2} / \mathrm{Vs}\right)$ \\
\hline $\mathrm{A}$ & $0 \%$ & $1.0 \times 10^{19}$ & 406 & 0.09 & 270 & 384 \\
$\mathrm{~B}$ & $0.10 \%$ & $5.3 \times 10^{18}$ & 101 & 0.09 & 688 & 151 \\
$\mathrm{C}$ & $0.20 \%$ & $7.6 \times 10^{18}$ & 114 & 0.13 & 563 & 127 \\
$\mathrm{D}$ & $0.23 \%$ & $7.8 \times 10^{18}$ & 61 & 0.12 & 1009 & 77 \\
$\mathrm{E}$ & $0.30 \%$ & $1.5 \times 10^{19}$ & 46 & 0.13 & 1346 & 53 \\
$\mathrm{~F}$ & $0.36 \%$ & $1.5 \times 10^{19}$ & 51 & 0.11 & 1555 & 55 \\
\hline \hline
\end{tabular}

$x$-dependence of the mobility in $n-\mathrm{GaAs}_{1-x} \mathrm{~N}_{x}$ with $x$ $<0.4 \%$ is controlled by the carrier lifetime rather than by the variation of $m_{e}^{*}$.

\section{EXPERIMENT}

Six Se-doped, $n$-type $\mathrm{GaAs}_{1-x} \mathrm{~N}_{x}$ epilayers $(0 \%<x$ $<0.4 \%$ ) were grown on (001) semi-insulating GaAs substrates by metal-organic molecular beam epitaxy. The growth conditions can be found elsewhere. ${ }^{22,23}$ Se was used as the $n$-type dopant instead of $\mathrm{Si}$ because $\mathrm{Si}$ and $\mathrm{N}$ passivate each other in Si-doped GaAsN due to the formation of $\mathrm{Si}-\mathrm{N}$ complexes. ${ }^{24}$ The $\mathrm{N}$ concentrations of the films (samples $\mathrm{A}-\mathrm{F}$ ), as determined by high-resolution $\mathrm{x}$-ray diffraction (HRXRD) measurements, ${ }^{25}$ are given in Table I. The thickness of the epilayers is in the $120-220 \mathrm{~nm} \mathrm{~nm}$ range, as obtained from the analysis of the HRXRD scans. Hall measurements at different temperatures were carried out in order to determine $N_{e}$ and the Hall mobility ( $\left.\mu_{\mathrm{Hall}}\right)$ of the layers. The third and fourth columns of Table I display $N_{e}$ and $\mu_{\text {Hall }}$ as obtained at $100 \mathrm{~K}$. Owing to the high $N_{e}$ values measured $\left(N_{e}>5 \times 10^{18} \mathrm{~cm}^{-3}\right)$, it can be assumed that the free carriers are degenerate in all the samples studied.

Raman measurements were performed at $80 \mathrm{~K}$ in a backscattering geometry on the (001) face. The spectra were obtained with the $514.5 \mathrm{~nm}$ line of an $\mathrm{Ar}^{+}$laser as excitation. Given that the penetration depth of the $514.5 \mathrm{~nm}$ light is appreciably smaller than the thickness of the GaAsN epilayers, the contribution of the GaAs substrate to the Raman signal is negligible. A Jobin-Yvon T64000 spectrometer equipped with a charge-coupled device detector was used to record the Raman measurements. The spectra were acquired with the high-resolution triple additive configuration of the spectrometer, with $100 \mu \mathrm{m}$ slits.

\section{RESULTS AND DISCUSSION}

Figure 1 shows $z(x y) \bar{z}$ Raman spectra of our Se-doped GaAsN layers (samples B-F). Three main features can be observed in all the spectra. The most intense peak is located at $\sim 294 \mathrm{~cm}^{-1}$ and corresponds to the GaAs-like LO phonon of GaAsN. ${ }^{26,27}$ In the case of $n$-type material, the LO peaks arise from the surface depletion region of the samples. ${ }^{14}$ The broad band centered at $\sim 268 \mathrm{~cm}^{-1}$ can be assigned to the GaAs-like TO mode of GaAsN. ${ }^{26,27}$ An additional broad feature that is not observed in undoped GaAsN and whose frequency and width change from sample to sample emerges between the TO and LO GaAs-like phonons. We assign this feature to a heavily damped LOPCM. Similar heavily damped LOPCM bands have been observed in $p$-GaAs, ${ }^{28}$ and in $n$-type InGaAs lattice matched to InP. ${ }^{19}$ While the LOPCMs in $p$-GaAs are heavily damped due to the very low hole mobility, Landau damping effects associated with the low electron effective mass of InGaAs are responsible for the low LOPCM lifetimes in this compound. In the case of $n$-type GaAsN, the observation of heavily damped LOPCMs instead of the well-known $L^{-}$and $L^{+}$coupled modes ${ }^{14}$ might be at first sight surprising. Note that, as expected, we observe both the $L^{+}$and $L^{-}$bands in the Raman spectra of our $n$-GaAs sample (inset of Fig. 1), while no $L^{+}$band is observed for any of the $n$-GaAsN epilayers.

To confirm that the features observed in the spectra of Fig. 1 correspond to a heavily damped LOPCM, we show in Fig. 2 the calculated LOPCM frequency versus $N_{e}$ for $n$-GaAs in the high damping regime (solid curve). This curve was obtained by calculating Raman line shapes at different $N_{e}$ values by using the fluctuation-dissipation formalism of Hon and Faust and the hydrodynamical (HD) model (see Refs. 16 and 29 for details), taking the maxima of the calculated LOPCM curves. For the sake of simplicity, we used for the calculations a constant value of $m_{\mathrm{opt}}^{*}=0.09 m_{e}$, which is the value found in heavily doped $n$-GaAs for $N_{e} \sim 1$

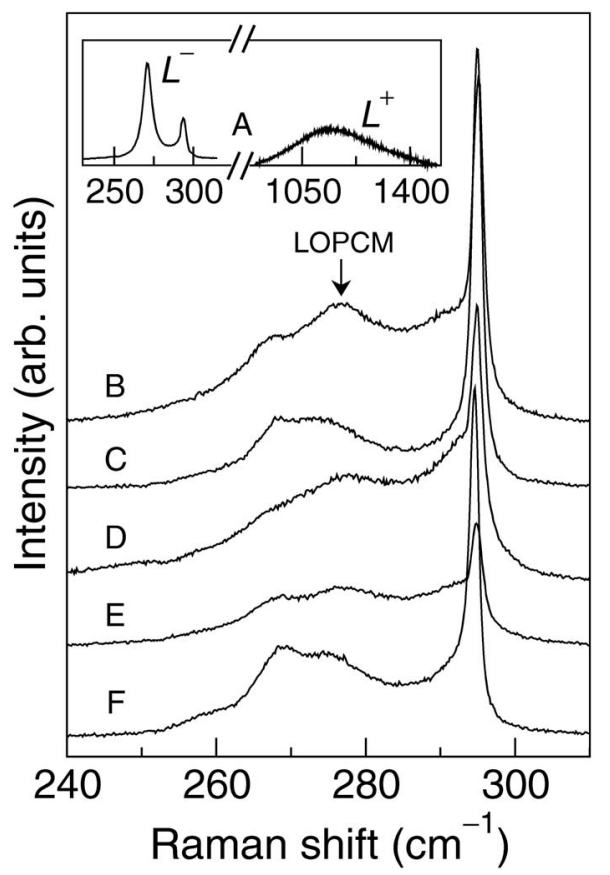

FIG. 1. Raman spectra acquired at $80 \mathrm{~K}$ of the $n$-GaAsN epilayers studied in this work (samples B-F). The inset shows the Raman spectrum of the $n$-GaAs epilayer (sample A). 


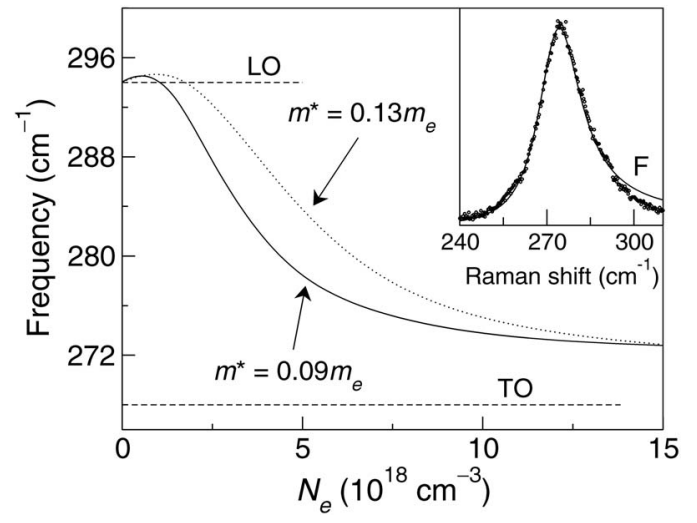

FIG. 2. Calculated $N_{e}$-dependence of the frequency of the heavily damped LOPCM in low mobility $n$-GaAs. The solid curve was calculated with $m_{\mathrm{opt}}^{*}=0.09 m_{e}$ and the dashed curve with $m_{\mathrm{opt}}^{*}=0.13 m_{e}$. Inset: LOPCM line shape fit (solid line) to the Raman spectrum of sample F (dots).

$\times 10^{19} \mathrm{~cm}^{-3} \cdot{ }^{20}$ A phenomenological electronic damping parameter $\Gamma_{e}=1000 \mathrm{~cm}^{-1}$, consistent with the low electron mobilities measured in our heavily doped samples (see Table I and Ref. 30), was used. The resulting curve confirms that, as for the case of $p$-GaAs, ${ }^{28}$ in $n$-GaAs with low electron mobility only a single LOPCM is expected between the TO and LO phonon modes of GaAs. In the case of GaAsN, the low (high) $\mu_{\text {Hall }}\left(\Gamma_{e}\right)$ values are a consequence of the N-related disorder. ${ }^{31,32}$ With regard to this, we would like to note that both $N_{e}$ and $\mu_{\text {Hall }}$ exhibited a weak temperature dependence in our samples. This suggests that the contribution of N-related alloy scattering to $\tau$ in these layers is much stronger than the contribution of other relaxation mechanisms such as inelastic phonon scattering.

The $L^{+}$mode observed in $n$-GaAs is particularly sensitive to variations in $N_{e}$ and in $m_{\mathrm{opt}}^{*}$. However, the heavily damped LOPCM does not display such high sensitivity. We therefore need to evaluate the effect of $m_{\mathrm{opt}}^{*}$ on the LOPCM frequency in order to determine whether the Raman spectra are sufficiently sensitive to effective mass variations, in which case valuable information about $m_{\text {opt }}^{*}$ can be obtained from the LOPCM spectra. Thus, we repeated the calculation of the frequency of the heavily damped LOPCM in $n$-GaAs as a function of $N_{e}$ by using a higher value of $m_{\mathrm{opt}}^{*}=0.13 m_{e}$. This is close to the values measured by Masia et al. in undoped $\mathrm{GaAs}_{1-x} \mathrm{~N}_{x}$ samples with $\mathrm{N}$ contents similar to those of our samples ${ }^{12}$ and also to the value obtained from magnetophonon resonance measurements in moderately doped GaAs $_{1-x} \mathrm{~N}_{x}$ with $x=0.1 \% .^{13}$

The resulting curve, plotted in Fig. 2 (dashed curve), shows that the frequency of the heavily damped LOPCM is higher (i.e., closer to the uncoupled LO mode) when $m_{\text {opt }}^{*}$ is increased. This result demonstrates that a change in $m_{\text {opt }}^{*}$ has measurable effects on the LOPCM frequency, and hence that the analysis of the Raman spectra can provide useful information about $m_{e}^{*}$ in dilute $n$-GaAsN.

To determine $m_{\mathrm{opt}}^{*}$ in our samples, we have carried out a line shape analysis of the Raman spectra based on the HD mode in the degenerate limit (see Refs. 16 and 29). The calculated line shapes are fitted to the experimental Raman spectra using the $N_{e}$ values obtained from Hall measure-

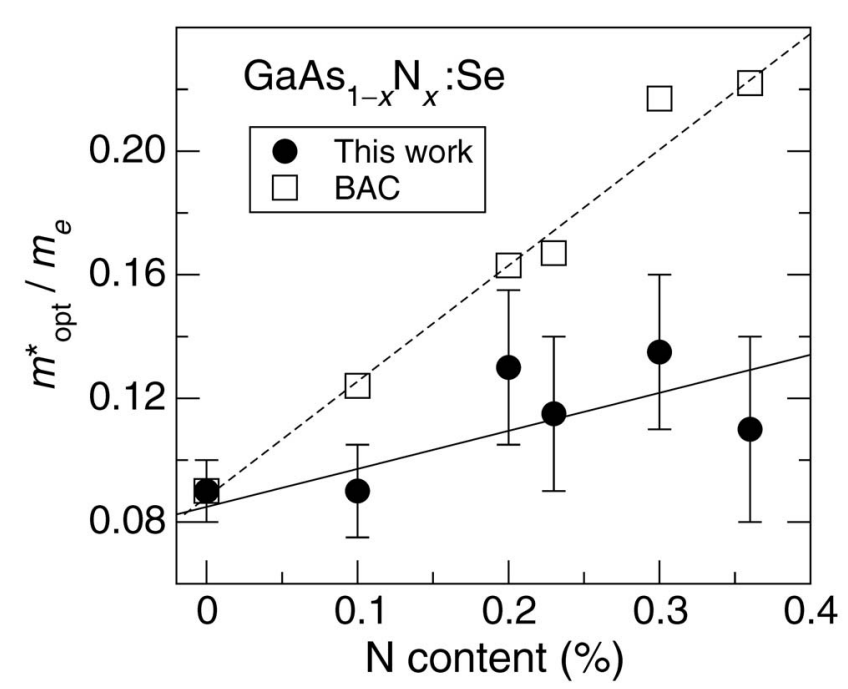

FIG. 3. Optical effective mass values, $m_{\mathrm{opt}}^{*}$, extracted from the analysis of the Raman measurements (dots) and values predicted by the two-level BAC model for the $\mathrm{N}$ composition and $N_{e}$ of the studied samples (open squares). The solid (dashed) line is intended only as a guide to the eye to show the increasing trend of $m_{\mathrm{opt}}^{*}$ with $\mathrm{N}$ content in our samples, as derived from the Raman spectra (BAC model).

ments (Table I) and leaving $m_{\mathrm{opt}}^{*}$ and $\Gamma_{e}$ as free parameters. Note that the lifetime $\tau$ of the LOPCMs, and hence the scattering time of the free carriers, is related to $\Gamma_{e}$ through the energy-time uncertainty relation $\tau^{-1}=2 \pi c \Gamma_{e}$. To avoid possible ambiguities in the fitting procedure, since different combinations of the fitting parameters may lead to satisfactory fits (see for instance Ref. 17 for the case of $p$-GaAs), we restrict $m_{\mathrm{opt}}^{*}$ and $\Gamma_{e}$ to vary within meaningful ranges, compatible with our measured Hall-effect carrier mobilities and with the $m_{e}^{*}$ values reported in the literature. ${ }^{12,13}$

We plot in the inset of Fig. 2 (solid line) the resulting line shape fit to the Raman spectrum of one of the analyzed $n$-GaAsN films (sample F). Note that prior to the fits the Raman peaks from the TO and $\mathrm{LO}$ phonon modes of GaAsN were fitted by Lorentzian line shapes and subtracted from the Raman spectra. Values of $m_{\mathrm{opt}}^{*}=0.11 m_{e}$ and $\Gamma_{e}=1555 \mathrm{~cm}^{-1}$ were extracted from this fit. As can be seen in the figure, the HD model provides good fits to the heavily damped LOPCM line shapes. We show in the fifth and sixth columns of Table I the $m_{\mathrm{opt}}^{*}$ and $\Gamma_{e}$ values obtained from the fits. In the particular case of the $n$-GaAs epilayer (sample A), $m_{\text {opt }}^{*}$ and $\Gamma_{e}$ were obtained from a fit to the $L^{+}$band. The last column of the table shows the mobility of the samples as estimated from $\tau=1 /\left(2 \pi c \Gamma_{e}\right)$ and $m_{\mathrm{opt}}^{*}$ with the expression $\mu_{\mathrm{Raman}}$ $=e \tau / m_{\mathrm{opt}}^{*}$. As can be seen in the table, both $\mu_{\text {Hall }}$ and $\mu_{\text {Raman }}$ tend to decrease with increasing $x$. Interestingly, a quite good agreement between the transport and the Raman mobilities is found, which provides support for the reliability of the Raman analysis.

In Fig. 3, we plot as a function of $x$ the $m_{\mathrm{opt}}^{*}$ values extracted from the fits for all the samples studied in this work (dots). Error bars have been drawn in the figure to take into account possible uncertainties in the determination by Halleffect measurements of $N_{e}$. These uncertainties also lead to significant relative errors in $\Gamma_{e}$, which we estimate to be around 10-20\%. For comparison, we also plot in Fig. 3 the 
$m_{\text {opt }}^{*}$ values predicted by the two-level BAC model for the $\mathrm{N}$ composition and $N_{e}$ of our samples (open squares). We use the analytical expressions for the effective mass given in Refs. 6 and 7, where the Fermi wave vector $k_{F}$ is evaluated in the degenerate limit as $k_{F}=\left(3 \pi^{2} N_{e}\right)^{1 / 3}$. To obtain the parameters that enter the $\mathrm{BAC}$ model at $80 \mathrm{~K}$, we extrapolate the values given in Ref. 33. As can be seen in Fig. 3, the $m_{\mathrm{opt}}^{*}$ values obtained with the BAC model increase as expected with increasing $x$. Note that the large $m_{\text {opt }}^{*}$ values obtained with the BAC model for the samples with the highest $\mathrm{N}$ content (samples $\mathrm{E}$ and $\mathrm{F}$, with $x=0.30$ and $0.36 \%$, respectively) are due to the high free-electron density of these two epilayers (see Table I). For such high doping levels, the BAC model predicts a large CB nonparabolicity, i.e., increased $m_{\text {opt }}^{*}$ values. As can be seen from the figure, however, for the $\mathrm{N}$ concentrations of the samples studied in this work, the $m_{\mathrm{opt}}^{*}$ values extracted from the LOPCM spectra are appreciably lower than those predicted by the two-level BAC model.

Although the discrepancy between the Raman data and the predictions of the two-level BAC model could be a consequence of intrinsic uncertainties in the analysis of the LOPCM spectra, it is quite likely that the BAC model offers a too simplistic description of the electron effective mass in GaAsN. The BAC model seems to correctly predict the large $\mathrm{N}$-induced increase of $m_{e}^{*}$ for $x>1 \%$ in $n$-type $\mathrm{In}_{y} \mathrm{Ga}_{1-y} \mathrm{As}_{1-x} \mathrm{~N}_{x}$. ${ }^{6}$ However, in the case of undoped GaAsN, magnetophotoluminescence measurements have revealed that $m_{e 0}^{*}$ is sizably higher than that predicted by the BAC model for the composition range of the samples studied here. These experimental results can be understood in terms of a description based on a linear combination of isolated nitrogen states, ${ }^{5}$ which shows that for the particular case of $0.1 \%<x<0.2 \%$ the CB-edge electronic states of $\mathrm{GaAs}_{1-x} \mathrm{~N}_{x}$ are strongly hybridized with $\mathrm{N}$ cluster states. Such a strong hybridization is responsible for the large $m_{e 0}^{*}$ values measured in this composition range. ${ }^{12}$ However, for heavily doped $n$ - $\mathrm{GaAs}_{1-x} \mathrm{~N}_{x}$ and the $\mathrm{N}$ compositions of the samples studied here, the Fermi energy lies well above the $\mathrm{N}$ cluster states (i.e., hundreds of meV depending on $x$ and $N_{e}$ ), and therefore only a weak interaction exists between the extended electronic states around the Fermi surface and the N-related localized cluster states. As a consequence, the enhancement of the electron effective mass due to the hybridization with $\mathrm{N}$ cluster states should play a much weaker role in our samples. These arguments can explain why, unlike the $m_{e 0}^{*}$ values measured by magnetophotoluminescence in undoped GaAsN, the $m_{\mathrm{opt}}^{*}$ values extracted from the LOPCM spectra are not larger than the values predicted by the twolevel BAC model. However, the fact that the Raman data are below the predictions of the BAC model (Fig. 3) remains unexplained. Further work is necessary to confirm these results and to ascertain how $m_{e}^{*}$ is affected by $N_{e}$.

Interestingly, the fact that $m_{\mathrm{opt}}^{*}$ is lower than predicted by the BAC model has important implications for the mechanisms responsible for the low electron mobility of heavily doped GaAsN. The $m_{\mathrm{opt}}^{*}$ values derived from our analysis, together with the $x$-dependence of $\mu_{\text {Hall }}$ and $\mu_{\text {Raman }}$, indicate that the electron mobility in GaAsN is limited more by the decrease of the electron scattering time with increasing $\mathrm{N}$ content as a consequence of N-related disorder than by changes of $m_{\mathrm{opt}}^{*}$ with increasing $\mathrm{N}$-content and/or carrier concentration.

\section{CONCLUSION}

We have found that $\mathrm{N}$-related disorder in $n-\mathrm{GaAs}_{1-x} \mathrm{~N}_{x}$ gives rise to a single heavily damped LOPCM even for $\mathrm{N}$ contents as low as $0.1 \%$. Despite the lower sensitivity to $N_{e}$ or $m_{e}^{*}$ variations of the heavily damped LOPCM in comparison to the $L^{+}$coupled-mode branch observed in $n$-GaAs, a careful analysis of the Raman spectra provides a means of estimating the optical effective mass of the free electrons, $m_{\text {opt }}^{*}$, in the $n-\mathrm{GaAs}_{1-x} \mathrm{~N}_{x}$ epilayers. We find that the $m_{\mathrm{opt}}^{*}$ values obtained from the fits are lower than those predicted by the BAC model in heavily doped $n$-GaAsN. These results suggest that the $x$-dependence of the measured electron mobility is mainly determined by the scattering time.

\section{ACKNOWLEDGMENTS}

This work is supported by the Spanish Government (Projects MAT 2004-0664 and MAT2007-63617, and Ramon y Cajal Program) and the EPSRC, United Kingdom.

${ }^{1}$ M. Henini, Dilute Nitride Semiconductors (Elsevier Science, Amsterdam, 2005)

${ }^{2}$ W. Shan, W. Walukiewicz, J. W. Ager III, E. E. Haller, J. F. Geisz, D. J. Friedman, and J. M. Olson and S. R. Kurtz, Phys. Rev. Lett. 82, 1221 (1999).

${ }^{3}$ J. D. Perkins, A. Mascarenhas, Y. Zhang, J. F. Geisz, D. J. Friedman, J. M. Olson, and S. R. Kurtz, Phys. Rev. Lett. 82, 3312 (1999).

${ }^{4}$ P. R. C. Kent and A. Zunger, Phys. Rev. Lett. 86, 2613 (2001).

${ }^{5}$ A. Lindsay and E. P. O’Reilly, Phys. Rev. Lett. 93, 196402 (2004).

${ }^{6}$ C. Skierbiszewski, P. Perlin, P. Wisniewski, W. Knap, T. Suski, W. Walukiewicz, W. Shan, K. M. Yu, J. W. Ager III, E. E. Haller, J. F. Geisz, and J. M. Olson, Appl. Phys. Lett. 76, 2409 (2000).

${ }^{7}$ C. Skierbiszewski, P. Perlin, P. Wisniewski, T. Suski, J. F. Geisz, K. Hingerl, W. Jantsch, D. E. Mars, and W. Walukiewicz, Phys. Rev. B 65, 035207 (2001).

${ }^{8}$ J. Endicott, A. Patanè, J. Ibáñez, L. Eaves, M. Bissiri, M. Hopkinson, R. Airey, and G. Hill, Phys. Rev. Lett. 91, 126802 (2003).

${ }^{9}$ J. Endicott, A. Patanè, D. Maude, L. Eaves, M. Hopkinson, and G. Hill, Phys. Rev. B 72, 041306 (2005)

${ }^{10}$ P. N. Hai, W. M. Chen, I. A. Buyanova, H. P. Xin, and C. W. Tun, Appl. Phys. Lett. 77, 1843 (2000).

${ }^{11}$ F. Masia, A. Polimeni, G. Baldassarri, M. Bissiri, M. Capizzi, P. J. Klar, and W. Stolz, Appl. Phys. Lett. 82, 4474 (2003).

${ }^{12}$ F. Masia, G. Pettinari, A. Polimeni, M. Felici, A. Miriametro, M. Capizzi, A. Lindsay, J. B. Healy, E. P. O’Reilly, A. Cristofoli, G. Bais, M. Piccin, S. Rubini, F. Martelli, A. Franciosi, P. J. Klar, K. Volz, and W. Stolz, Phys. Rev. B 73, 073201 (2006).

${ }^{13}$ G. Allison, S. Spasov, A. Patanè, L. Eaves, A. Ignatov, D. K. Maude, M. Hopkinson, and R. Airey, Phys. Rev. B 75, 115325 (2007).

${ }^{14}$ G. Abstreiter, M. Cardona, and A. Pinczuk, in Light Scattering in Solids $I V$, edited by M. Cardona and G. Güntherodt (Springer-Verlag, Berlin, 1984), Vol. 54.

${ }^{15}$ R. Cuscó, J. Ibáñez, and L. Artús, Phys. Rev. B 57, 12197 (1998).

${ }^{16}$ L. Artús, R. Cuscó, J. Ibáñez, N. Blanco, and G. Gonzalez-Diaz, Phys. Rev. B 60, 5456 (1999).

${ }^{17}$ W. Limmer, M. Glunk, S. Mascheck, A. Koeder, D. Klarer, W. Schoch, K. Thonke, R. Sauer, and A. Waag, Phys. Rev. B 66, 205209 (2002).

${ }^{18}$ P. A. Grandt, A. E. Griffith, M. O. Manasreh, D. J. Friedman, S. Dogan, and D. Johnstone, Appl. Phys. Lett. 85, 4905 (2004).

${ }^{19}$ R. Cuscó, L. Artús, S. Hernández, J. Ibáñez, and M. Hopkinson, Phys. Rev. B 65, 035210 (2001).

${ }^{20}$ H. R. Chandrasekhar and A. K. Ramdas, Phys. Rev. B 21, 1511 (1980).

${ }^{21}$ M. Cardona, Phys. Rev. B 121, 752 (1961).

${ }^{22}$ I. Suemune, K. Uesugi, and T. Y. Seong, Semicond. Sci. Technol. 17, 755 
(2002).

${ }^{23}$ K. Uesugi and I. Suemune, Appl. Phys. Lett. 79, 3284 (2001).

${ }^{24}$ K. M. Yu, W. Walukiewicz, J. Wu, D. E. Mars, D. R. Chamberlin, M. A. Scarpulla, O. D. Dubon, and J. F. Geisz, Nat. Mater. 1, 185 (2002).

${ }^{25}$ K. Uesugi, N. Morroka, and I. Suemune, Appl. Phys. Lett. 74, 1254 (1999).

${ }^{26}$ T. Prokofyeva, T. Sauncy, M. Seon, M. Holtz, Y. Qiu, S. Nikishin, and H. Temkin, Appl. Phys. Lett. 73, 1409 (1998).

${ }^{27}$ J. Ibáñez, E. Alarcón-Lladó, R. Cuscó, L. Artús, and M. Hopkinson, J. Appl. Phys. 102, 013502 (2007).
${ }^{28}$ K. Wan and J. F. Young, Phys. Rev. B 41, 10772 (1990).

${ }^{29}$ J. Ibáñez, R. Cuscó, and L. Artús, Phys. Status Solidi B 223, 715 (2001).

${ }^{30}$ M. Ramsteiner, J. Wagner, P. Hiesinger, K. Köhler, and U. Rössler, J. Appl. Phys. 73, 5023 (1993).

${ }^{31}$ S. Fahy, A. Lindsay, H. Ouerdane, and E. P. O'Reilly, Phys. Rev. B 74, 035203 (2006).

${ }^{32}$ M. P. Vaughan and B. K. Ridley, Phys. Rev. B 75, 195205 (2007).

${ }^{33}$ A. Grau, T. Passow, and M. Hetterich, Appl. Phys. Lett. 89, 202105 (2006). 